\title{
Opportunistic osteoporosis screening: contrast-enhanced dual-layer spectral CT provides accurate measurements of vertebral bone mineral density
}

\author{
Ferdinand Roski ${ }^{1}$ (1) Johannes Hammel ${ }^{1,2} \cdot$ Kai Mei $^{1,3} \cdot$ Bernhard Haller $^{4} \cdot$ Thomas Baum $^{5} \cdot$ Jan S. Kirschke ${ }^{5}$ \\ Daniela Pfeiffer ${ }^{1} \cdot$ Klaus Woertler $^{1}$ - Franz Pfeiffer ${ }^{2} \cdot$ Peter B. Noël $^{3} \cdot$ Alexandra S. Gersing ${ }^{1} \cdot$ Benedikt J. Schwaiger $^{1,5}$
}

Received: 2 July 2020 / Revised: 4 August 2020 / Accepted: 17 September 2020 / Published online: 14 October 2020

(C) The Author(s) 2020

\begin{abstract}
Objectives Osteoporosis remains under-diagnosed, which may be improved by opportunistic bone mineral density (BMD) measurements on CT. However, correcting for the influence of intravenous iodine-based contrast agent is challenging. The purpose of this study was to assess the diagnostic accuracy of iodine-corrected vertebral BMD measurements derived from nondedicated contrast-enhanced phantomless dual-layer spectral CT (DLCT) examinations.

Methods Vertebral volumetric DLCT-BMD was measured in native, arterial, and portal-venous scans of 132 patients (63 \pm 16 years; $32 \%$ women) using virtual monoenergetic images (50 and $200 \mathrm{keV})$. For comparison, conventional BMD was determined using an asynchronous QCT calibration. Additionally, iodine densities were measured in the abdominal aorta (AA), inferior vena cava, and vena portae (VP) on each $\mathrm{CT}$ phase to adjust for iodine-related measurement errors in multivariable linear regressions and a generalized estimated equation, and conversion equations were calculated.

Results BMD values derived from contrast-enhanced phases using conversion equations adjusted for individual vessel iodine concentrations of VP and/or AA showed a high agreement with those from non-enhanced scans in Bland-Altman plots. Mean absolute errors (MAE) of DLCT-BMD were $3.57 \mathrm{mg} / \mathrm{ml}$ for the arterial $\left(R^{2}=0.989\right)$ and $3.69 \mathrm{mg} / \mathrm{ml}$ for the portal-venous phase $\left(R^{2}=0.987\right)$ (conventional BMD: $4.70\left[R^{2}=0.983\right]$ and $\left.5.15 \mathrm{mg} / \mathrm{ml}\left[\mathrm{R}^{2}=0.981\right]\right)$. In the phase-independent analysis, MAE was $4.49 \mathrm{mg} / \mathrm{ml}$ for DLCT $\left(R^{2}=0.989\right)$ (conventional BMD: $4.82 \mathrm{mg} / \mathrm{ml}\left[R^{2}=0.981\right]$ ).

Conclusions Converted BMD derived from contrast-enhanced DLCT examinations and adjusted for individual vessel iodine concentrations showed a high agreement with non-enhanced DLCT-BMD, suggesting that opportunistic BMD measurements are feasible even in non-dedicated contrast-enhanced DLCT examinations.

Key Points

- Accurate BMD values can be converted from contrast-enhanced DLCT scans, independent from the used scan phase.

- DLCT-BMD measurements from contrast-enhanced scans should be adjusted with iodine concentrations of portal vein and/or abdominal aorta, which significantly improves the goodness-of-fit of conversion models.
\end{abstract}

Keywords Bone density $\cdot$ Contrast media $\cdot$ Osteoporosis · Tomography, X-ray computed

Ferdinand Roski

ferdinand.roski@tum.de

1 Department of Radiology, Klinikum rechts der Isar, School of Medicine, Technical University of Munich, Ismaninger Str. 22, 81675 Munich, Germany

2 Biomedical Physics \& Munich School of BioEngineering, Technical University of Munich, 85748 Garching, Germany
3 Department of Radiology, Perelman School of Medicine, University of Pennsylvania, Philadelphia, PA 19104, USA

4 Institute of Medical Statistics and Epidemiology, Klinikum rechts der Isar, School of Medicine, Technical University of Munich, 81675 Munich, Germany

5 Department of Neuroradiology, Klinikum rechts der Isar, School of Medicine, Technical University of Munich, 81675 Munich, Germany 


$\begin{array}{ll}\text { Abbreviations } \\ \text { AA } & \text { Aorta abdominalis } \\ \text { ADT } & \text { Androgen deprivation therapy } \\ \text { AR } & \text { Arterial scan phase } \\ \text { BMD } & \text { Bone mineral density } \\ \text { cBMD } & \text { Converted BMD } \\ \text { CE } & \text { Contrast-enhanced } \\ \text { CTDI } & \text { CT dose index } \\ \text { DECT } & \text { Dual-energy computed tomography } \\ \text { DLCT } & \text { Dual-layer spectral computed tomography } \\ \text { DXA } & \text { Dual-energy x-ray absorptiometry } \\ \text { GEE } & \text { Generalized estimated equations } \\ \text { HA } & \text { Calcium hydroxyapatite } \\ \text { IVC } & \text { Inferior vena cava } \\ \text { MAE } & \text { Mean absolute error } \\ \text { MDCT } & \text { Multidetector computed tomography } \\ \text { PI } & \text { Phase-independent } \\ \text { PV } & \text { Portal-venous scan phase } \\ \text { QCT } & \text { Quantitative computed tomography } \\ \text { R }{ }^{2} \text { c } & \text { Change of } R^{2} \\ \text { VFFs } & \text { Vertebral fragility fractures } \\ \text { VMI } & \text { Virtual monoenergetic image } \\ \text { VOI } & \text { Volume of interest } \\ \text { VP } & \text { Vena portae } \\ & \end{array}$

\section{Introduction}

Osteopenia and osteoporosis remain a severe challenge in health care, not only from a clinical perspective - the treatment gap is estimated at $59 \%$ of women and $57 \%$ of men in the EU [1] - but also from an epidemiologic view considering its contribution to an increasing number of fall-related deaths [2]. Bearing in mind that the majority of the causal vertebral fragility fractures (VFFs) already occurs in osteopenic individuals [3] emphasizes the importance of early detecting patients at risk. A UK audit, however, showed that incidental VFFs are substantially underdiagnosed in non-dedicated CT exams due to deficient reporting [4]. Here, even basic tools (e.g., routine sagittal reformations, stringent terminology) could improve clinico-radiological workflow and patients' outcome [5]. Besides, fracture-related treatment costs represent the main cost factor for osteoporosis, thus causing considerable economic burden to health systems [1].

A diagnostic gap was not only found in patients with primary or postmenopausal osteoporosis [6-8] - this is also an issue regarding patients with secondary causes for reduced bone mineral density (BMD), e.g., patients suffering from malignant conditions and undergoing therapy also affecting BMD [9]. For instance, men with prostate cancer receiving androgen deprivation therapy (ADT) bear an increased risk to suffer from accelerated bone loss, which is a major adverse effect [10-12]. Even though guidelines recommend evaluation with baseline and periodic follow-up BMD quantification [13], there is a severe under-use of dedicated imaging methods such as dual-energy $\mathrm{x}$-ray absorptiometry (DXA) or quantitative CT (QCT) - e.g., for patients with non-metastatic prostate cancer, only about one in ten patients over 65 years receiving ADT undergoes a baseline bone densitometry [14-16].

Most patients with such malignant conditions are regularly subjected to contrast-enhanced CT for (re-)staging or the assessment of age-related comorbidities, in which a vast set of imaging data is generated. This gave rise to the question whether contrast-enhanced CT scans may be used for opportunistic BMD measurements to avoid additional dedicated examinations.

In clinical osteodensitometry, dual-energy CT (DECT) imaging has been available for more than 30 years [17]. However, in contrast to other DECT approaches such as dual-source $\mathrm{CT}$ or single-source $\mathrm{CT}$ with rapid $\mathrm{kV}$ switching, which are routinely performed in single-energy mode, DLCT continuously detects dual-energy information in standard CT protocols. Consequently, this novel implementation of DECT provides spectral data on clinical demand in all examinations without the need to prospectively select qualified patients for DECT imaging. Providing spectral information retrospectively in all examinations not only does facilitate radiological workflow, but also virtually lends itself for the retrospective approach of opportunistically measuring BMD.

By means of two superimposed detector layers, dual-layer spectral CT (DLCT) enables the separation of low- and highenergy photons, therefore providing energy-specific attenuation coefficients of present materials [18]. Based on this spectral information, one can infer on the material composition of body tissues or fluids, e.g., by measuring the concentration of calcium hydroxyapatite for BMD quantification [19] or the iodine concentration for quantifying contrast enhancement [20].

In a previous publication, the feasibility of opportunistic screening for osteoporosis was already demonstrated in vivo for native DLCT examinations [21]. Consequently, the scope of applicability in this paper is confined to clinical settings in which current non-enhanced DLCT data are missing.

The objective of this study was to evaluate the usability of contrast-enhanced DLCT scans for calculating precise BMD values based on non-dedicated CT examinations, i.e., with examinations performed for indications other than osteodensitometry.

For this purpose, the present analysis investigated (I) the effects of iodine contrast agent on the accuracy of DLCTBMD measurements and (II) any possible improvement of accuracy by adjusting for concentrations of iodine contrast agent measured in large vessels. 


\section{Materials and methods}

\section{Patient population}

According to the study protocol, 471 consecutive patients with a complete triphasic DLCT examination of the abdomen or thorax/abdomen were enrolled if exams were performed for indications other than osteodensitometry. Of those, patients with metal-containing implants adjacent to the thoracolumbar spine such as aortic stent grafts $(n=293)$ or spinal instrumentation $(n=36)$ were excluded. The same applies for patients with malignant conditions affecting the spine, e.g., spinal bone metastases $(n=7)$ or hematologic diseases $(n=3)$, resulting in a patient sample of 132 patients ( $63 \pm 16$ years; $32 \%$ women) who were retrospectively identified in the institutional PACS between September 2016 and October 2018.

Institutional Review Board approval was obtained prior to this study (Ethics Commission of the School of Medicine, Technical University of Munich, Germany). Informed consent was waived for this retrospective analysis of routinely acquired imaging data.

\section{DLCT image acquisition}

CT images were acquired with one DLCT scanner (IQon Spectral CT, Philips Healthcare). For all scans, a routine abdominal CT protocol was used with a tube voltage of $120 \mathrm{keV}$. Non-contrast-enhanced (NE) scans had an exposure of $107 \pm$ $49 \mathrm{mAs}($ mean $\pm \mathrm{SD})$ and a mean CT dose index $\left(\mathrm{CTDI}_{\mathrm{vol}}\right)$ of $9.7 \pm 4.5 \mathrm{mGy}$; contrast-enhanced (CE) scans had an exposure of $110 \pm 43 \mathrm{mAs}$ and a mean CTDI $\mathrm{vol}_{\text {of }}$ of $10.0 \pm 3.9 \mathrm{mGy}$.

$\mathrm{CE}$ scans were performed using iomeprol, a non-ionic iodinated contrast agent for intravenous application (Imeron 400 $\mathrm{MCT}$, Bracco Imaging Deutschland $\mathrm{GmbH})$. Per routine clinical protocol and depending on the clinical situation, a volume of 50 to $70 \mathrm{ml}$ was administered with a flow rate of $3 \mathrm{ml} / \mathrm{s}$. Arterial phases were triggered when the average CT numbers of a volume of interest (VOI) in the descending thoracic aorta exceeded a threshold value of $150 \mathrm{HU}$. The portal-venous phases started $70 \mathrm{~s}$ after contrast administration.

\section{Image analysis, post-processing, and BMD calculation}

On sagittal reformations, circular VOIs were manually placed in the ventral halves of L1 to L3. If one or more of these showed a pathology such as a fracture or extensive degenerative changes, adjacent thoracolumbar vertebrae were used instead. CT numbers in NE, arterial (AR), and portal-venous (PV) phases were extracted from both conventional and two virtual monoenergetic images at different energy levels (VMI; 50 and $200 \mathrm{keV}$ ), respectively (Fig. 1).

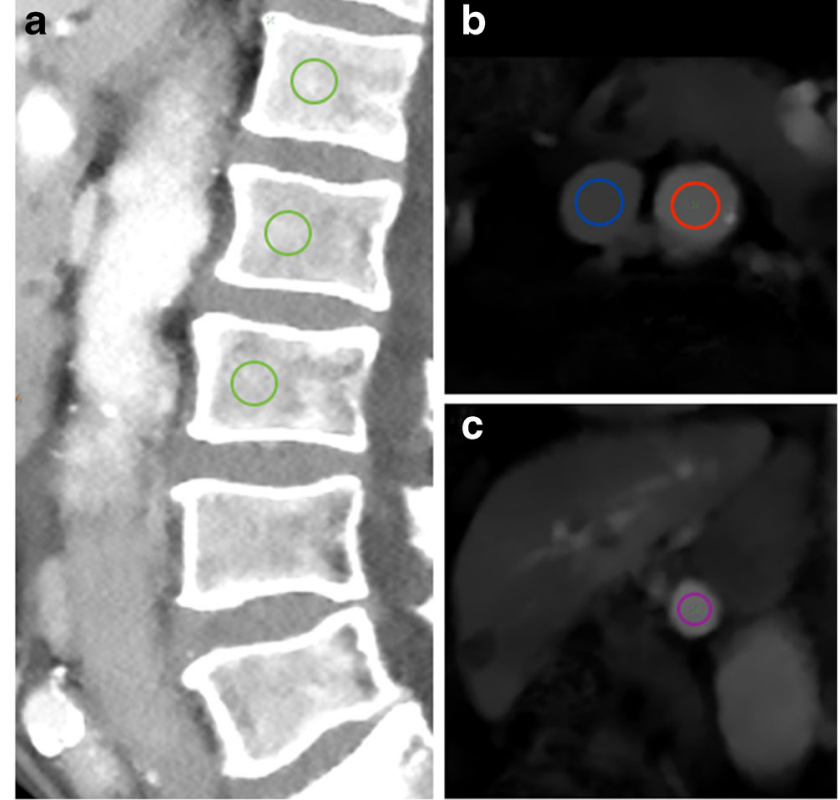

Fig. 1 Sagittal (a), axial (b), and multiplanar reformation (c) of dual-layer spectral CT imaging of a 77-year-old male patient. Spinal VOIs are positioned in the ventral halves of L1-L3 (green) (VMI at $50 \mathrm{keV}$ ). Vascular VOIs are positioned orthogonally and mid-luminally in the abdominal aorta (red), the inferior vena cava (blue), and the portal vein (violet) (iodine density maps)

Subsequently, patients' BMD values were calculated in two ways: Based on VMIs, DLCT-BMD values were computed for all three scan phases as previously described by Roski et al [21]. In short, after performing an ex vivo highdose calibration scan of three hydroxyapatite (HA)-containing insets of the European spine phantom (ESP) with DLCT, attenuation values of VMIs can be linearly assigned to respective $\mathrm{BMD}$ values via projection calibration as they are proportional to measured HA concentrations.

Conventional BMD values were similarly calculated for all scan phases by using an asynchronous calibration $[22,23]$ with attenuation values of a standard QCT phantom (Mindways QCT) consisting of five differently concentrated solutions of $\mathrm{K}_{2} \mathrm{PO}_{4}$. Here, phantom measurements were averaged over 33 examinations, which were performed in the same time period and on the same scanner. Instead of VMIs, conventional images were used for BMD quantification. These are reconstructed by using a weighted sum of counts measured in the two detector layers, with resulting images being analogous to a conventional single-energy CT scanner [24].

Additionally, mid-luminal iodine concentrations $(\mathrm{mg} / \mathrm{ml})$ were measured within the abdominal aorta (AA) and the inferior vena cava (IVC) on axial images - immediately inferior to the renal vessels - as well as within the vena portae (VP) on multiplanar reformations (Fig. 1). A commercially available spectral CT software was used for the generation of iodine density maps based on iodine-water decomposition (IntelliSpace Portal 10.1.0, Philips Healthcare). 


\section{Statistical analysis}

Both DLCT and conventional BMD values from CE scans were separately correlated with their reference values from corresponding NE scans in multivariable linear regression models using forward selection. BMD values from CE scans, vessel iodine concentrations (AA, IVC, and VP), age, and sex represented the set of selectable independent variables. DLCT-BMD from NE scans had previously shown its validity in a comparison with QCT [21]. Consequently, these data served as standard of reference for the contrast-enhanced DLCT-BMD values in this study. Likewise, the respective native values served as the dependent variable for conventional BMD. This linear regression analysis was performed separately for AR and PV. For the phase-independent (PI) approach, due to present within-subject correlation, generalized estimated equations (GEE) were used to investigate functional relations between contrast-enhanced and native BMD.

Beforehand, the patient sample was randomly split up into a training cohort $(n=88)$ for the multivariable linear regression model and a test cohort $(n=44)$ for the eventual validation of its predictive accuracy. For the phase-independent GEE analysis, individual patient scans of the AR and PV phase were independently assigned to the training cohort $(n=176)$ and the test cohort $(n=88)$.

Bland-Altman plots showing data from the test cohorts were used to examine the agreement of native and calculated BMD values, thus estimating the predictive value of the regression model. Moreover, agreement was calculated on a patient base as mean of absolute errors (MAEs) before and after the application of conversion equations. The external data of the test cohort was used to validate the conversion equations of both linear regressions and the GEE analysis with $R^{2}$ values.

The statistical calculations were performed using SPSS 23 (IBM).

\section{Results}

\section{In vivo DLCT-based and conventional BMD measurements}

For DLCT-BMD, measurements in native scans averaged to $102.95 \pm 46.33 \mathrm{mg} / \mathrm{ml}$ (mean $\pm \mathrm{SD})$, whereas arterial $(112.23$ $\pm 7.88 \mathrm{mg} / \mathrm{ml}[+9.0 \%])$ and portal-venous $(126.86 \pm$ $53.89 \mathrm{mg} / \mathrm{ml}[+23.2 \%])$ scan phases revealed substantially higher results (Fig. 2). DLCT-BMD from AR and PV showed high correlations ( $r=0.994$ [95\% confidence interval, 0.991$0.996]$ and $r=0.989$ [0.984-0.992]), yet a low agreement with DLCT-BMD from NE.

Conventionally calculated BMD showed similar, yet continuously lower results: native BMD values were 97.00 \pm $43.48 \mathrm{mg} / \mathrm{ml}$, whereas in CE phases, likewise, BMD values were substantially higher with $106.44 \pm 44.35 \mathrm{mg} / \mathrm{ml}(+9.7 \%)$ for $\mathrm{AR}$ and $119.67 \pm 42.93 \mathrm{mg} / \mathrm{ml}(+23.3 \%)$ for $\mathrm{PV}$, respectively.

\section{Linear regression and generalized estimating equation analyses}

Both phase-specific regression models had very high coefficients of determination for DLCT-BMD measurements with conventional BMD values consistently showing lower goodness of fit (Table 1).

Besides respective BMD values from CE scan phases, a significant association of the iodine concentration of the VP could be detected for all three DLCT models (AR: change of $R^{2}\left(R^{2} \mathrm{c}\right)=0.007$ (DLCT-BMD) $/ R^{2} \mathrm{c}=0.008$ (conventional BMD), PV: $R^{2} \mathrm{c}=0.001 /$ not significant). Patient's age was significantly associated with the outcome only for the phaseindependent GEE analysis, the iodine concentration of AA only for the PV linear regression model $\left(R^{2} \mathrm{c}=0.008 / R^{2} \mathrm{c}=\right.$
Fig. 2 Scatter plot showing DLCT-BMD values of 132 patients (averaged over three vertebrae each) derived from different contrast-enhanced scan phases $(\mathrm{AR}=$ red and $\mathrm{PV}=$ violet $)$; the black bisecting line serves as standard of reference showing the corresponding line of native BMD values

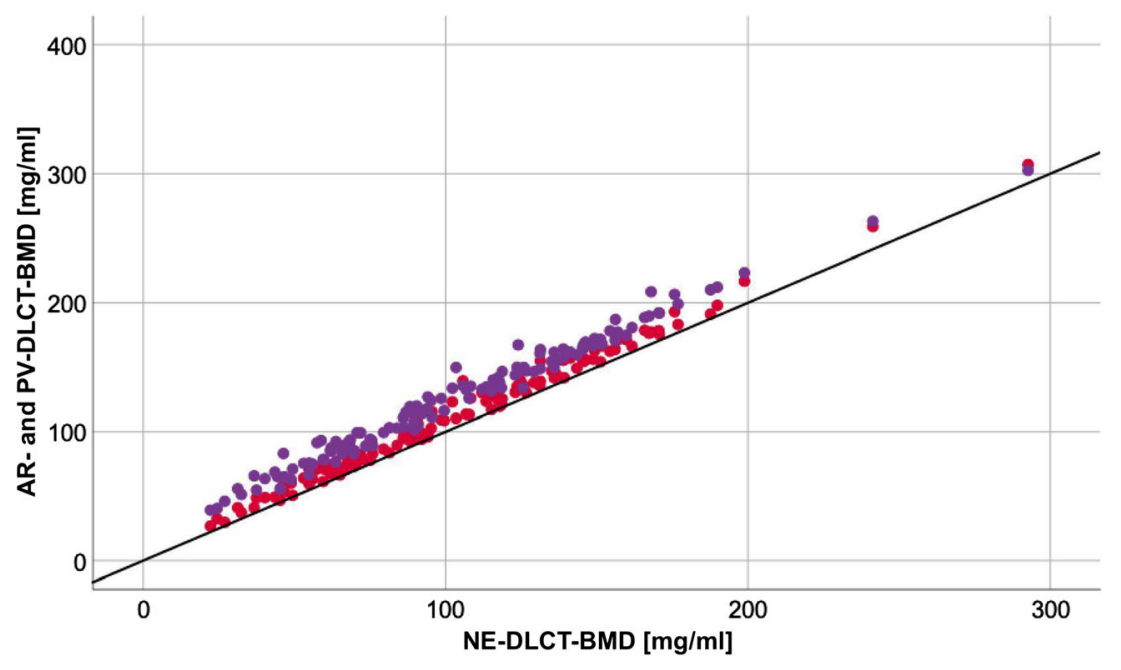


Table 1 Comparison of $R^{2}$ change and adjusted $R^{2}$ for several multivariable linear regression analyses with forward selection, either based on contrast-enhanced DLCT-BMD or on conventional BMD data, respective native BMD values served as dependent variable, illustrated for AR and PV

\begin{tabular}{llll}
\hline & Variable & DLCT-BMD $\left(R^{2} \mathrm{c} / R^{2}\right.$ adj $)$ & Conv. BMD $\left(R^{2} \mathrm{c} / R^{2}\right.$ adj $)$ \\
\hline $\begin{array}{c}\text { a) arterial phase } \\
\text { AR }\end{array}$ & & \\
1 & BMD $_{\mathrm{AR}}$ & $0.983(p=0.000)$ & $0.978(p=0.000)$ \\
2 & Iodine $_{\mathrm{VP}}$ & $0.007(p=0.000)$ & $0.008(p=0.000)$ \\
Total & & 0.990 & 0.986 \\
b) portal-venous phase & & \\
PV & & & $0.966(p=0.000)$ \\
1 & BMD $_{\mathrm{PV}}$ & $0.976(p=0.000)$ & $0.010(p=0.000)$ \\
2 & Iodine $_{\mathrm{AA}}$ & $0.008(p=0.000)$ & - \\
3 & Iodine $_{\mathrm{VP}}$ & $0.001(p=0.019)$ & 0.975 \\
Total & & 0.984 & \\
\hline
\end{tabular}

0.010). Neither iodine concentration of IVC nor sex was identified as a significant predictor for any scan phase.
The derived conversion equations for the DLCT-BMD data are:

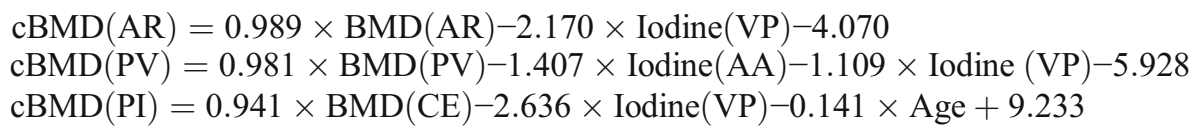

\section{Agreement of native and calculated BMD}

After converting the BMD values of the test cohorts using the presented equations, corresponding Bland-Altman plots showed a substantial agreement between converted and respective natively measured BMD (Fig. 3).

For the arterial phase, DLCT-BMD had a mean of absolute errors (MAEs) of 9.99 (95\% confidence interval, 8.38-11.59) $\mathrm{mg} / \mathrm{ml}$ before and $3.57(2.56-4.58) \mathrm{mg} / \mathrm{ml}$ after the conversion of measured to estimated BMD, whereas conventional BMD improved from $9.93(8.12-11.75)$ to $4.70(3.53-5.86) \mathrm{mg} / \mathrm{ml}$.

For the portal-venous phase, conversion of contrastenhanced data reduced MAE from $21.58(19.73-23.42) \mathrm{mg} / \mathrm{ml}$ to $3.69(2.69-4.70) \mathrm{mg} / \mathrm{ml}$ for DLCT-BMD (21.76 [19.39$24.13]$ to 5.15 [3.76-6.54] mg/ml for conventional BMD).

The MAE of the phase-independent GEE model changed from 14.57 (12.81-16.33) $\mathrm{mg} / \mathrm{ml}$ before to 4.49 (3.87-5.11) $\mathrm{mg} / \mathrm{ml}$ after the conversion of DLCT-BMD (14.77 [12.88$16.66]$ to 4.82 [3.99-5.64] $\mathrm{mg} / \mathrm{ml}$ for conventional BMD).

The above conversion equations were validated with external data of the test cohorts and consistently showed high coefficients of determination (Table 2).

\section{Discussion}

Converted BMD values, derived from routine clinical contrast-enhanced DLCT and adjusted for vessel iodine concentrations, showed a high agreement with non-enhanced DLCT-BMD. Moreover, the phase-independent conversion equation provides results which are adequate for the detection of low BMD in a clinical context as well.

Before this conversion, DLCT-BMD values were consistently higher for all scan phases when measured in contrastenhanced scans compared with their native BMD references (Fig. 2). This represents the challenge of adequately separating intravascular iodine within the vertebra from $\mathrm{HA}$, which is attributable to similar spectral absorption behavior of the two components. Another explanation for this BMD variation is the present dual-layer set-up, which cannot provide absolute selectivity on the detector level due to an overlap of the highand low-energy spectra $[25,26]$.

As the very same VOIs were used for comparing both calculations, the utilization of spectral information is capable of additionally improving overall BMD accuracy. Although DLCT is still not perfectly specific for HA, the consistently higher coefficients of determination in the linear regression (Table 1) and the GEE analysis suggest that DLCT-BMD shows a more pronounced functional relation between contrast-enhanced and native scans compared with conventional BMD. Besides, quantifying iodine concentrations outside osseous structures, more precisely in large vessels (Vena portae, Aorta), significantly improves the accuracy of converted BMD.

In a pilot analysis $(n=12)$, tissue iodine concentrations of paraspinal muscle and fat were measured for all scan phases. Balancing the linear model between completeness and 

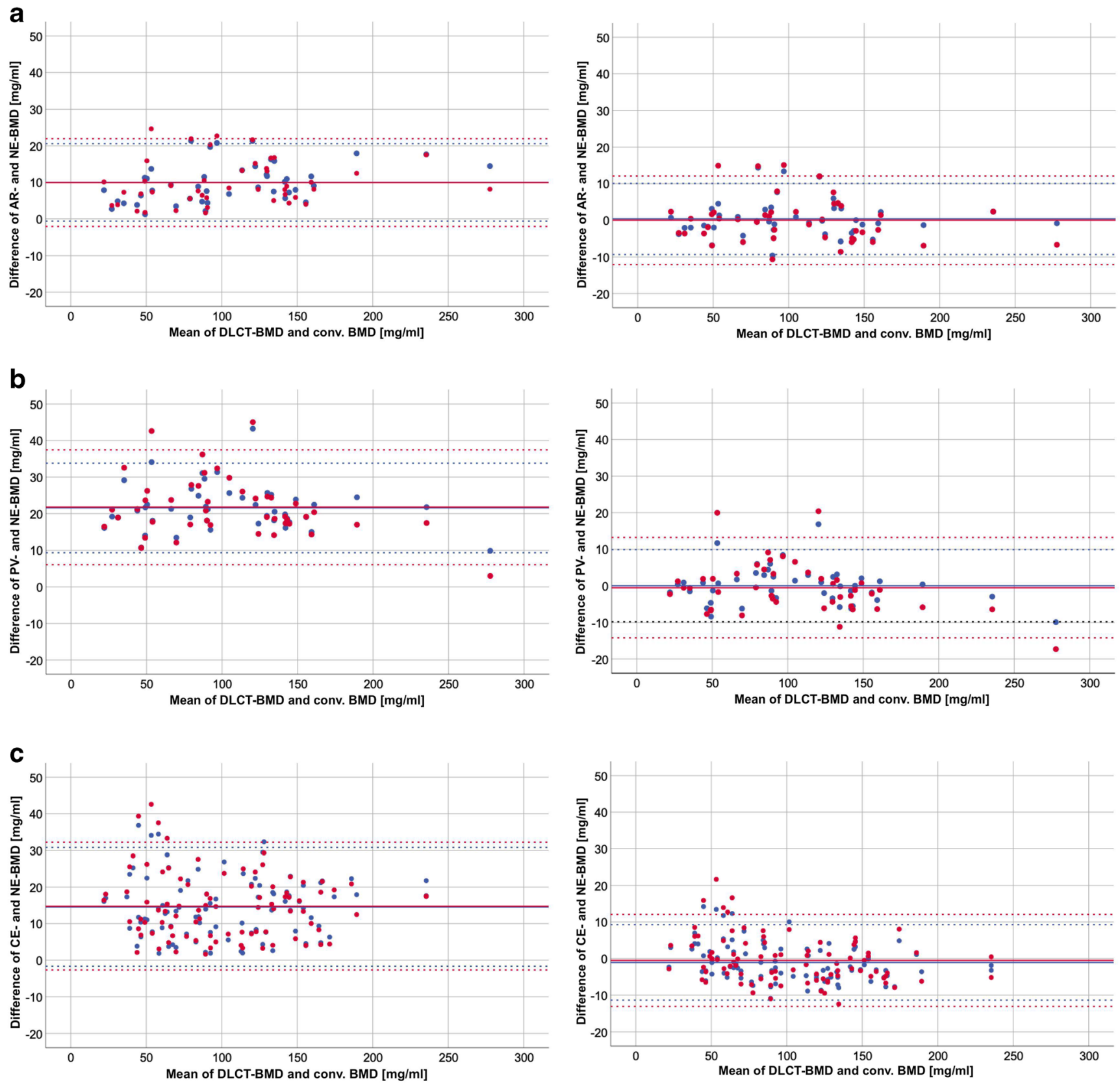

Fig. 3 Bland-Altman plots showing data of randomly selected test cohorts ( $n=44$ for $\mathrm{AR}$ and $\mathrm{PV}, n=88$ for $\mathrm{PI})$; the $y$-axis shows differences between BMD derived from contrast-enhanced scan phases and native BMD; the $x$-axis shows mean values of native DLCT-BMD and native conventional BMD; the black solid lines indicate equivalence of BMD values; the colored solid lines indicate the mean of BMD

Table 2 Coefficients of determination $\left(R^{2}\right)$ for external data of the test cohorts ( $n=44$ for AR and PV, $n=88$ for PI)

\begin{tabular}{lll}
\hline & DLCT-BMD $\left(R^{2}\right)$ & Conv. BMD $\left(R^{2}\right)$ \\
\hline Arterial phase (AR) & 0.989 & 0.983 \\
Portal-venous phase (PV) & 0.987 & 0.981 \\
Phase-independent analysis (PI) & 0.989 & 0.981 \\
\hline
\end{tabular}

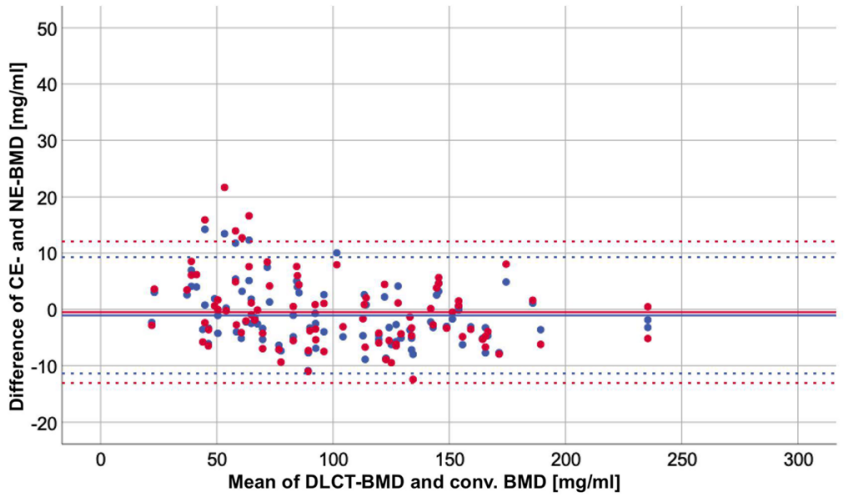

differences; the colored dotted lines indicate the $95 \%$ limits of agreement (mean difference $\pm 1.96 \mathrm{SD}$ ); blue represents data of DLCT-BMD; red represents data of conventional $\mathrm{BMD}$; top row $=\mathrm{AR}(\mathbf{a})$, middle row $=$ PV (b), bottom row = PI (c); left column: BMD data before application of conversion equation, right column: estimated BMD after application of conversion equation

complexity, however, the rationale was to set up a slender, numerically stable model without overfitting or multicollinearity. Here, preliminary data suggested focusing on vessel iodine measurements in order to adjust for the influence of intravascular contrast agent. After the conversion, DLCT-BMD showed a high agreement between native and converted values in Bland-Altman plots (Fig. 3a-c) and a substantial reduction of MAEs (relative reduction of $64 \%$ for 
$\mathrm{AR}, 83 \%$ for PV, and $69 \%$ for PI). The validation with external data of the test cohort revealed coefficients of determination that are equivalent to those of the training cohort (Table 2). This finding confirms a numerically stable model for BMD prediction that is accurate in new data.

Particularly the phase-independent approach features critical advantages over phase-specific conversion equations as in clinical routine, the iodine concentration within the vertebra's trabecular compartment may be affected by numerous factors apart from scan timing [27, 28]: e.g., contrast application speed, volume, the patient's circulatory capacity, volume of distribution, red vs. yellow bone marrow ratio. BMD values derived from a phase-independent conversion equation could minimize the influence of those factors and therefore may be most useful in clinical reality.

Focusing on contrast-enhanced clinical DLCT exams, these routinely acquired clinical CT data were utilized for opportunistic BMD measurements to monitor changes such as tumor- or therapy-associated bone loss, when current nonenhanced scans are missing. The proposed DLCT-based method provides the opportunity to retrospectively screen for low BMD and could at once spare patients additional exposure to radiation by dedicated bone densitometric exams. Quantifying individual iodine concentrations in abdominal vessels turned out to be a practicable way of adjusting for the influence of intravascular contrast agent. In this context, there is extensive literature indicating the high accuracy of DLCT-based iodine quantification: within the typically encountered concentration ranges in clinical radiology, relative mean errors of about 3.3 to $4.6 \%$ were found, with simulated patient size and tube voltage inconsistently affecting measuring precision $[20,29,30]$.

Comparable studies assessing iodine-associated effects on BMD quantification with multidetector CT (MDCT) encountered limitations: a study by Baum et al had a small training cohort for the conversion equation, relied on phantom calibration and only used PV scans [31]. A comparable study by Kaesmacher et al was limited by a small number of enrolled patients [32].

The conversion equations in this DLCT study, however, are based on a solid training cohort of 88 patients. An additional correction step was introduced by adjusting for vascular iodine concentrations, which can be obtained with minimal effort in work and time. Besides, DLCT potentially combines the inherent benefits of dual-energy imaging for osteodensitometry with the major advantage of QCT, i.e., exclusive volumetric measurements of the trabecular compartment, which is more sensitive regarding therapy-associated bone remodeling processes [33] — however, without needing synchronous phantom calibration.

Note that the present statistical models already show very high determination coefficients for conventional BMD: contrary to a comparable study investigating asynchronously calibrated BMD derived from contrast-enhanced MDCT, DLCT-BMD has substantially narrower $95 \%$ limits of agreement $(-10$ to $+11 \mathrm{mg} / \mathrm{ml}$ (DLCT) vs. ca. -30 to $+14 \mathrm{mg} / \mathrm{ml}$ (MDCT) for AR and -10 to $+10 \mathrm{mg} / \mathrm{ml}$ (DLCT) vs. ca. -39 to $+8 \mathrm{mg} / \mathrm{ml}$ for PV) and a better linear fit $\left(R^{2}: 0.983\right.$ vs. 0.923 for $\mathrm{AR}, 0.976$ vs. 0.904 for $\mathrm{PV}$ ) [32]. Considering the minor contribution of the vessel iodine corrections, these results suggest a notably higher measuring accuracy of the DLCT scanner compared with MDCT.

Apart from several phantom studies, two in vivo trials already showed the diagnostic accuracy of native DLCT regarding osteodensitometric applications: Van Hedent et al demonstrated that DLCT-based BMD measurements perform very well in the detection of decreased BMD using DXA as standard of reference [34]. A previously mentioned study by Roski et al showed that non-enhanced DLCT-based BMD measurements are on a par with phantom-based QCT [21].

This study has limitations. As CT exams were retrieved from clinical routine, there was no systematic variation in the amount of applied contrast agent to adjust for contrast load. Furthermore, neither overall circulatory parameters nor the local vascularization of the vertebral bodies for correlating contrast distribution could be investigated according to the retrospective nature of this analysis. Moderating both scan protocol inconsistencies and varying circulatory parameters, the phase-independent analysis is potentially meeting clinical reality best. Besides, the vertebral VOIs were placed manually, which contributes to the risk of a higher intra- or interobserver variability. A next step would be to overcome this issue by implementing a BMD analysis pipeline drawing on automatic segmentation. Additional longitudinal studies will be needed to investigate the in vivo reproducibility and the predictive power regarding incidental fractures.

In summary, this study showed that BMD values can be accurately estimated from contrast-enhanced multiphasic dual-layer spectral CT examinations, even independently from the used contrast phase. Moreover, measuring only one abdominal vessel for iodine concentration could significantly increase the goodness-of-fit in statistical models. Therefore, iodine-adjusted DLCT-BMD measurements suggest their potential value for a reliable opportunistic assessment of BMD even in routine clinical contrast-enhanced examinations.

Funding Open Access funding enabled and organized by Projekt DEAL. This work was supported by the European Research Council (ERC-StG2014 637164; H2020, AdG 695045); by the German Federal Ministry of Education and Research (BMBF) under grant IMEDO (13GW0072C); by the German Research Foundation (as part of the Gottfried Wilhelm Leibniz program); and by the TUM Institute for Advanced Study, funded by the German Excellence Initiative.

\section{Compliance with ethical standards}

Guarantor The scientific guarantor of this publication is Dr. Benedikt J. Schwaiger, MD. 
Conflict of interest The authors of this manuscript declare no relationships with any companies, whose products or services may be related to the subject matter of the article.

Statistics and biometry No complex statistical methods were necessary for this paper.

Informed consent Written informed consent was waived by the Institutional Review Board.

Ethical approval Institutional Review Board approval was obtained.

\author{
Methodology \\ - retrospective \\ - diagnostic or prognostic study \\ - performed at one institution
}

Open Access This article is licensed under a Creative Commons Attribution 4.0 International License, which permits use, sharing, adaptation, distribution and reproduction in any medium or format, as long as you give appropriate credit to the original author(s) and the source, provide a link to the Creative Commons licence, and indicate if changes were made. The images or other third party material in this article are included in the article's Creative Commons licence, unless indicated otherwise in a credit line to the material. If material is not included in the article's Creative Commons licence and your intended use is not permitted by statutory regulation or exceeds the permitted use, you will need to obtain permission directly from the copyright holder. To view a copy of this licence, visit http://creativecommons.org/licenses/by/4.0/.

\section{References}

1. Hernlund E, Svedbom A, Ivergard M et al (2013) Osteoporosis in the European Union: medical management, epidemiology and economic burden. A report prepared in collaboration with the International Osteoporosis Foundation (IOF) and the European Federation of Pharmaceutical Industry Associations (EFPIA). Arch Osteoporos 8:136

2. Sanchez-Riera L, Carnahan E, Vos T et al (2014) The global burden attributable to low bone mineral density. Ann Rheum Dis 73:16351645

3. Pasco JA, Seeman E, Henry MJ, Merriman EN, Nicholson GC, Kotowicz MA (2006) The population burden of fractures originates in women with osteopenia, not osteoporosis. Osteoporos Int 17: 1404-1409

4. Howlett DC, Drinkwater KJ, Mahmood N, Illes J, Griffin J, Javaid K (2020) Radiology reporting of osteoporotic vertebral fragility fractures on computed tomography studies: results of a UK national audit. Eur Radiol. https://doi.org/10.1007/s00330-020-06845-2

5. Abrahamsen B (2020) Hiding in plain sight-unreported osteoporotic vertebral fractures. Eur Radiol. https://doi.org/10.1007/s00330020-06944-0

6. Feldstein A, Elmer PJ, Orwoll E, Herson M, Hillier T (2003) Bone mineral density measurement and treatment for osteoporosis in older individuals with fractures: a gap in evidence-based practice guideline implementation. Arch Intern Med 163:2165-2172

7. Siris ES, Miller PD, Barrett-Connor E et al (2001) Identification and fracture outcomes of undiagnosed low bone mineral density in postmenopausal women: results from the National Osteoporosis Risk Assessment. JAMA 286:2815-2822

8. Lafata JE, Kolk D, Peterson EL et al (2007) Improving osteoporosis screening: results from a randomized cluster trial. J Gen Intern Med 22:346-351

9. Pfeilschifter J, Diel IJ (2000) Osteoporosis due to cancer treatment: pathogenesis and management. J Clin Oncol 18:1570-1593

10. Melton LJ 3rd, Lieber MM, Atkinson EJ et al (2011) Fracture risk in men with prostate cancer: a population-based study. J Bone Miner Res 26:1808-1815

11. Dalla Via J, Daly RM, Owen PJ, Mundell NL, Rantalainen T, Fraser SF (2019) Bone mineral density, structure, distribution and strength in men with prostate cancer treated with androgen deprivation therapy. Bone 127:367-375

12. Greenspan SL, Coates P, Sereika SM, Nelson JB, Trump DL, Resnick NM (2005) Bone loss after initiation of androgen deprivation therapy in patients with prostate cancer. J Clin Endocrinol Metab 90:6410-6417

13. Gralow JR, Biermann JS, Farooki A et al (2013) NCCN Task Force report: bone health in cancer care. J Natl Compr Canc Netw 11(Suppl 3):S1-S50 quiz S51

14. Holt A, Khan MA, Gujja S, Govindarajan R (2015) Utilization of bone densitometry for prediction and administration of bisphosphonates to prevent osteoporosis in patients with prostate cancer without bone metastases receiving antiandrogen therapy. Cancer Manag Res 7:13-18

15. Suarez-Almazor ME, Peddi P, Luo R, Nguyen HT, Elting LS (2014) Low rates of bone mineral density measurement in Medicare beneficiaries with prostate cancer initiating androgen deprivation therapy. Support Care Cancer 22:537-544

16. Shahinian VB, Kuo YF (2013) Patterns of bone mineral density testing in men receiving androgen deprivation for prostate cancer. J Gen Intern Med 28:1440-1446

17. Genant HK, Boyd D (1977) Quantitative bone mineral analysis using dual energy computed tomography. Invest Radiol 12:545551

18. Rassouli N, Etesami M, Dhanantwari A, Rajiah P (2017) Detectorbased spectral CT with a novel dual-layer technology: principles and applications. Insights Imaging 8:589-598

19. Mei K, Schwaiger BJ, Kopp FK et al (2017) Bone mineral density measurements in vertebral specimens and phantoms using duallayer spectral computed tomography. Sci Rep 7:17519

20. Pelgrim GJ, van Hamersvelt RW, Willemink MJ et al (2017) Accuracy of iodine quantification using dual energy CT in latest generation dual source and dual layer CT. Eur Radiol 27:39043912

21. Roski F, Hammel J, Mei K et al (2019) Bone mineral density measurements derived from dual-layer spectral CT enable opportunistic screening for osteoporosis. Eur Radiol. https://doi.org/10.1007/ s00330-019-06263-Z

22. Wang L, Su Y, Wang Q et al (2017) Validation of asynchronous quantitative bone densitometry of the spine: accuracy, short-term reproducibility, and a comparison with conventional quantitative computed tomography. Sci Rep 7:6284

23. Budoff MJ, Malpeso JM, Zeb I et al (2013) Measurement of phantomless thoracic bone mineral density on coronary artery calcium CT scans acquired with various CT scanner models. Radiology 267:830-836

24. Hojjati M, Van Hedent S, Rassouli N et al (2017) Quality of routine diagnostic abdominal images generated from a novel detectorbased spectral CT scanner: a technical report on a phantom and clinical study. Abdom Radiol (NY) 42:2752-2759 
25. Shefer E, Altman A, Behling R et al (2013) State of the art of CT detectors and sources: a literature review. Curr Radiol Rep 1:76-91

26. Faby S, Kuchenbecker S, Sawall S et al (2015) Performance of today's dual energy CT and future multi energy CT in virtual non-contrast imaging and in iodine quantification: a simulation study. Med Phys 42:4349-4366

27. Acu K, Scheel M, Issever AS (2014) Time dependency of bone density estimation from computed tomography with intravenous contrast agent administration. Osteoporos Int 25:535-542

28. Pompe E, Willemink MJ, Dijkhuis GR, Verhaar HJ, Mohamed Hoesein FA, de Jong PA (2015) Intravenous contrast injection significantly affects bone mineral density measured on CT. Eur Radiol $25: 283-289$

29. Ehn S, Sellerer T, Muenzel D et al (2018) Assessment of quantification accuracy and image quality of a full-body dual-layer spectral CT system. J Appl Clin Med Phys 19:204-217

30. Sauter AP, Kopp FK, Munzel D et al (2018) Accuracy of iodine quantification in dual-layer spectral CT: influence of iterative reconstruction, patient habitus and tube parameters. Eur J Radiol 102: 83-88
31. Baum T, Muller D, Dobritz M, Rummeny EJ, Link TM, Bauer JS (2011) BMD measurements of the spine derived from sagittal reformations of contrast-enhanced MDCT without dedicated software. Eur J Radiol 80:e140-e145

32. Kaesmacher J, Liebl H, Baum T, Kirschke JS (2017) Bone mineral density estimations from routine multidetector computed tomography: a comparative study of contrast and calibration effects. J Comput Assist Tomogr 41:217-223

33. Black DM, Greenspan SL, Ensrud KE et al (2003) The effects of parathyroid hormone and alendronate alone or in combination in postmenopausal osteoporosis. N Engl J Med 349:1207-1215

34. Van Hedent S, Su KH, Jordan DW et al (2018) Improving bone mineral density assessment using spectral detector CT. J Clin Densitom. https://doi.org/10.1016/j.jocd.2018.10.004

Publisher's note Springer Nature remains neutral with regard to jurisdictional claims in published maps and institutional affiliations. 